Publications of the Astronomical Society of the Pacific 109: $707-718,1997$ June

\title{
A Wide-Bandwidth Digital Recording System for Radio Pulsar Astronomy
}

\author{
F. A. JENET, W. R. COOK, T. A. PRINCE, AND S. C. UNWIN \\ Division of Physics, Mathematics, and Astronomy, California Institute of Technology, Mail Code 220-47, Pasadena, \\ California 91125 \\ Electronic mail: merlyn@srl.caltech.edu \\ Received 1996 November 12; accepted 1997 February 21
}

\begin{abstract}
The study of radio pulsars at the highest time resolution is currently limited by the capability of the signal detection system to accept a wide-bandwidth signal, and to sample the data rapidly enough. We describe a new instrument for pulsar research which utilizes baseband recording at $400 \mathrm{Mbit} \mathrm{s}^{-1}$ to achieve both a high bandwidth and a high sustained data rate. The wide bandwidth digital recording (WBDR) system is based on a custom analog/digital VLSI digitizer operating at $50 \mathrm{MHz}$, and a commercial digital cassette tape recorder. Signal analysis is performed entirely in software, using a massively parallel computer. Since we record a representation of the electric vector of the pulsar emission, the instrument is very flexible, and the data can be analyzed in several modes in software. We can synthesize the software equivalent of a conventional hardware filterbank, and we have implemented a "coherent dedispersion" algorithm, which yields a sample time of $10 \mathrm{~ns}$. The combination of a wide bandwidth and sustained data rate make this instrument a unique and powerful tool for pulsar astronomy. Our instrument is particularly well-suited to searches for millisecond pulsars at low frequency, in directions where pulsed signals are strongly dispersed by the interstellar medium. We present results based on dual-polarization test observations in a $600 \pm 25 \mathrm{MHz}$ band at the Owens Valley Radio Observatory 40-m Telescope.
\end{abstract}

\section{INTRODUCTION}

Radio pulsars are rotating highly magnetized neutron stars that emit periodic bursts of broadband radio noise. The emission typically has a steep power-law spectrum with a turnover frequency of order $100 \mathrm{MHz}$. Pulse periods range from $1.6 \mathrm{~ms}$ to a few seconds, with typical on-pulse duty cycles of a few percent. Pulsars are studied for a variety of purposes in astrophysics and fundamental physics (Taylor and Stinebring 1986). Millisecond pulsars are of particular interest for understanding neutron star physical properties, emission mechanisms, and the evolution of binary systems (Phinney and Kulkarni 1994; Bhattacharya 1992).

Searching for new millisecond pulsars represents a challenge for radio observations, because they are often faint (time-averaged flux densities of $1 \mathrm{mJy}$ or less), requiring long integration times for detection, and hence large datasets for search algorithms. The interstellar medium imprints its signature on the pulsar emission through dispersion, scattering, and Faraday rotation. In particular, dispersion in the interstellar medium produces a frequencydependent delay to the pulsed emission, and is usually the most significant of these effects, especially at low frequency. This delay is proportional to the dispersion measure, which is the total column density of electrons along the line of sight to the source: $\mathrm{DM}=\int n_{e} d l$; in this paper we quote $\mathrm{DM}$ in units of $\mathrm{pc} \mathrm{cm}^{-3}$. For instance, for an observing frequency $f_{0}=0.6 \mathrm{GHz}$, and dispersion measure $\mathrm{DM}=50$, the broadening (width of an initial $\delta$ function) across a $B=50 \mathrm{MHz}$ band, is $96 \mathrm{~ms}$-many times the period of a millisecond pulsar. Therefore, instrumenta- tion for detection and study of pulsars must be able to correct for the effects of dispersion.

Interstellar scattering produces a broadened pulse profile due to multipath propagation effects. It represents a fundamental limitation to the time resolution which can be achieved, since its effects cannot be removed with pulsar instrumentation. The magnitude of the effect is dependent on the distribution of scattering material along the line of sight, which is usually uncertain. Models of the interstellar medium have been developed which predict the DM distribution on the sky, and the size of the pulse "tail" due to scattering ( Taylor and Cordes 1993). At $0.6 \mathrm{GHz}$, the tail is in the range $\sim 100-1000 \mu \mathrm{s}$, for $\mathrm{DM} \sim 50$; this is a significant limitation in searches for millisecond pulsars at low frequency (broadening $\propto \nu^{-4.4}$ ) (Backer 1988). Cordes et al. (1985) have used pulse structure as a probe of the interstellar medium.

Conventional pulsar instrumentation includes filterbank and autocorrelation spectrometers. These spectrometers reduce dispersion effects by effectively dividing the wideband signal into many narrow frequency channels. In a filterbank system, the radio frequency (RF) signal is typically downconverted to an intermediate frequency (IF) and then multiplexed into a set of $n$ filters which divide the original signal into $n$ signals of bandwidth $B / n$. If $n$ is sufficiently large, the dispersion delay across the individual filter channels is small. The outputs of the filters are typically passed through a square-law detector, averaged over an appropriate number of time samples, digitized, then recorded. The data are analyzed by selecting a dispersion measure, introducing relative delays in the individual filterbank channels to com- 
pensate for the dispersion delays, and summing the power outputs of individual frequency channels. The Princeton Mark III pulsar timing system (Stinebring et al. 1992) is one of several systems based on filterbanks; it "folds" the filterbank data at the pulse period to accumulate a pulse profile in each channel.

Autocorrelation spectrometers are similar in approach, with the difference that the IF signal is mixed to baseband, then digitized at the Nyquist frequency corresponding to the signal bandwidth, after which the average autocorrelation as a function of lag is computed digitally and the results recorded. Power versus frequency is then obtained by taking the fast Fourier transform (FFT) of the autocorrelation function. Incoherent dedispersion can then be applied in the same manner as described above. The Caltech FPTM (Navarro 1994) is an example of an autocorrelation spectrometer.

The instrument described here takes a third, and fundamentally different approach. We record the baseband radio signal using a high-speed digital tape recorder, and perform all of the analysis in software. This approach provides great flexibility, since our raw data are a representation of the electric vector of the incoming pulsar signal. It converts the process of data acquisition and analysis from primarily hardware oriented to one in which the major steps are done in software. We can emulate a filterbank or autocorrelator in software, but with the advantage that the number of filter channels, or autocorrelator lags, becomes a parameter that can be chosen for each observation, rather than a fundamental feature of the design.

Perhaps the most important difference is the ability to correct almost exactly for the effects of dispersion using "coherent dedispersion" (Hankins and Rickett 1975), thereby regaining (except for the fundamental limitation due to multipath scattering) the minimum possible (Nyquist) sample time $t_{\text {sam }}=1 /(2 B)$. Note that for a filterbank, the corresponding sample time is $n /(2 B)$. This correction is described in more detail in Sec. 3. The ability to achieve very rapid time sampling of a dispersed signal is essential for a number of pulsar studies. Likewise, access to the raw voltage data allows us to develop much more sophisticated tools for dealing with radio-frequency interference (RFI).

The concept of baseband recording is not new (Hankins 1971; Hankins and Rickett 1975; Hankins et al., 1987). However, such systems have in the past been limited in capability, primarily because the bandwidth of the sampled signal was restricted to a manageable size. Our widebandwidth digital recording (WBDR) system is the first such instrument to utilize a continuous bandwidth comparable with those of filterbanks, and to record this band at a sustained rate. Wietfeldt et al. 1995 have developed a similar recording system based on the S2 VLBI recorder. The Berkeley Pulsar Processor developed at UC Berkeley (Backer 1995) performs coherent dedispersion in hardware, after dividing the band into several narrower channels.

In Sec. 2 of this paper, we describe the instrumentation we have developed for radio pulsar astronomy. Section 3 discusses the playback and analysis of pulsar data. Since the data reduction is computationally intensive, we focus on algorithms developed to run on massively parallel computers. We describe in Sec. 4 the performance of the system, and some representative early results from observations at the Owens Valley Radio Observatory 40-m Telescope. In Sec. 5 we discuss future directions for pulsar research with this class of instrumentation.

\section{INSTRUMENTATION}

\subsection{System Overview}

The basic design philosophy behind the WBDR system is to convert much of the task of observing and detecting radio pulsars from primarily a hardware problem to one in which the major steps are done in software. At the telescope, the hardware serves to collect and represent, in digital form, the incoming electric field of the pulsar radiation. A high-speed commercial tape recorder is used to store the data, and a similar machine is used to play back data into a massively parallel computer for signal analysis.

This approach has several advantages for pulsar astronomy, as described in the introduction. We therefore opted to keep the telescope hardware as simple as possible, concentrating the features and flexibility for a variety of pulsar projects in the analysis software. This simplicity enabled us to develop a prototype system in only a few months, and to test it with the Owens Valley Radio Observatory 40-m telescope, operated by Caltech.

A small number of observational parameters suffice to specify data collection for the baseband recording system. The key parameters are: total bandwidth $B$, number of polarizations $n_{p}$, oversampling factor $\eta$, and number of bits per sample $n_{b}$. Since we complex sample the input data stream, the total data rate $R$ is given by $R=B \eta n_{p} n_{b}$. (Note: for real sampled data, also known as Nyquist sampled data, $R=2 B \eta n_{p} n_{b}$. ) The WBDR system operates at the sustained data rates now supported by the fastest digital tape recorders. Earlier recording systems of this type were limited to very narrow bandwidths.

With the constraints in mind, we set the following design goals: (1) record as high a data rate as practical; (2) retain hardware flexibility in data rate, signal quantization levels, number of channels, etc., without adding complexity; (3) keep the amount of custom hardware to a minimum, allowing quick progress to a working prototype.

We selected one of the fastest commercially available digital tape recorders, the Datatape Inc. LP-400, which operates at up to $R=400 \mathrm{Mbit} \mathrm{s}^{-1}$ using ANSI D-1 format video cassettes. This rate exceeds by more than an order of magnitude the rates of commonly used computer tape drives. It features highly reliable recording, achieved by sophisticated error checking and recovery. This reliability allows simplification of data time tagging (see below): Accurate timing is essential for certain projects. The ability of the Datatape recorder to play back at $1 / 8$ of the record speed greatly improves its flexibility in interfacing with workstation-class computers.

A significant consideration for this project is the cost of tape media, since a fundamental feature of our system is that we cannot average the raw data at all. However, the 

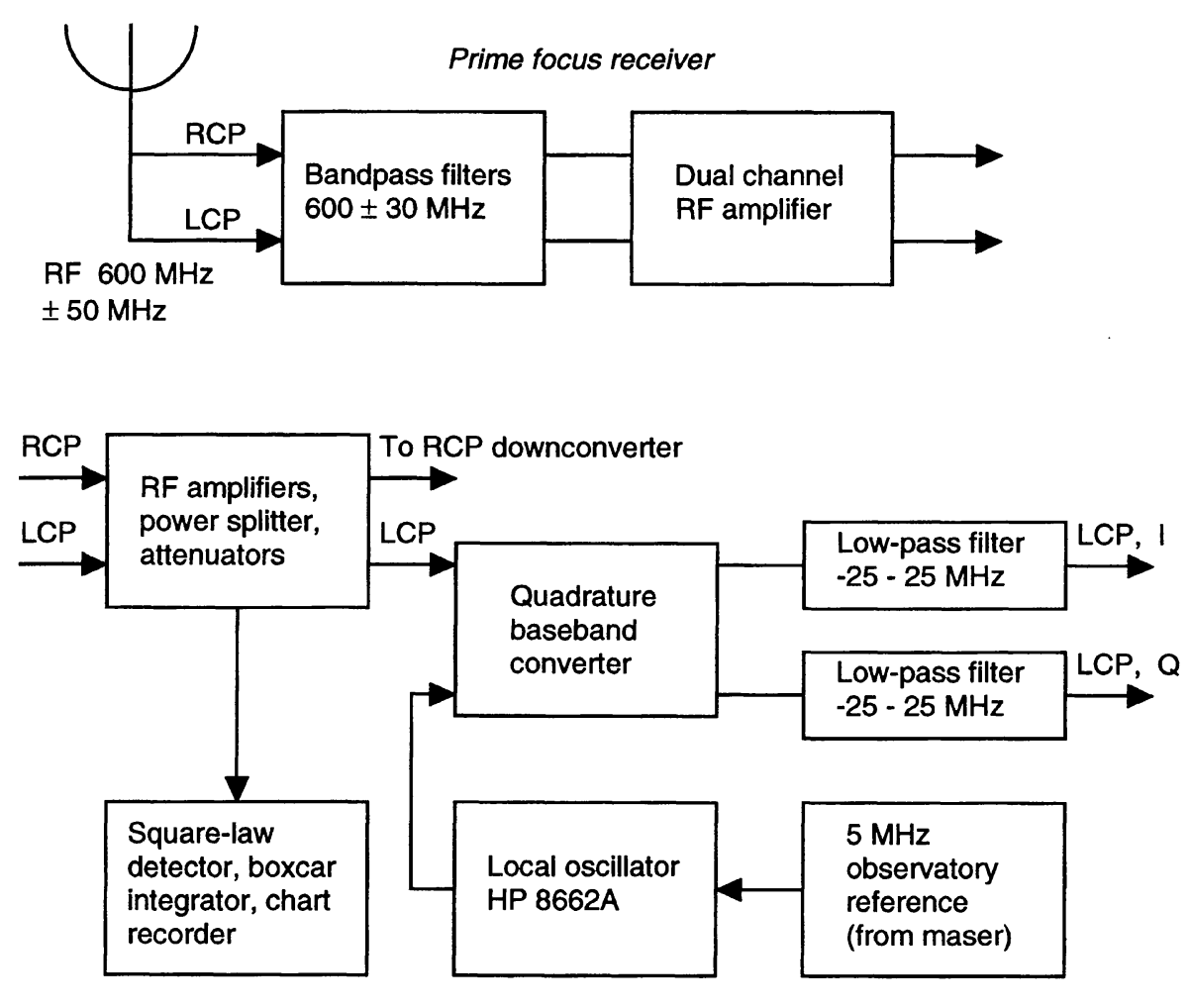

Downconverter rack (control room)

FIG. 1-Analog signal path for the system as installed at the OVRO 40-m Telescope in 1994 October, for observation at $600 \mathrm{MHz}$. A second quadrature downconverter (not shown) is used for the orthogonal polarization.

cost per byte of D-1 tape is slightly cheaper than for the 5-Gbyte Exabyte format. The uninterrupted scan length is $32 \mathrm{~min}$ at the maximum data rate; this is set by the 96 Gbyte capacity of the longest available tape. Tape changing takes $\lesssim 60 \mathrm{~s}$, and timing information is preserved across the gap, allowing multiple-tape scans to be linked.

The signal flow from the telescope receiver output to the tape recorder requires the following steps:

(1) Mixing the receiver RF or IF band to baseband, low-pass filtering, and amplifying to the level required by the digitizers.

(2) Sampling the analog voltage at a precisely controlled rate, and digitizing with a specified number of bits.

(3) Inserting synchronization and timing information into the data stream.

(4) Accumulating statistics of data to verify correct digitization of the analog input.

(5) Converting signal levels and impedance as necessary to drive the tape recorder input buffer. This step is done using standard CMOS/ECL converter chips.

All but the first and last steps are performed in a custom CMOS VLSI chip, described below.

The IF section (Fig. 1) comprises analog hardware to amplify the IF (or RF) signals from the telescope receiver, downconvert in frequency to baseband, and low-pass filter to a bandwidth appropriate for the digitizer clock rate. This is the only part of the system which is telescope specific.

We used a conventional complex $(\mathrm{I}, \mathrm{Q})$ mixer with baseband low-pass filters, using discrete commercial components. The analog performance is determined by amplitude and phase matching of the $90^{\circ}$ hybrid coupler, mixers, and filters. The hybrid couplers operate over about a one-octave frequency range, allowing us to select any observing band within the receiver passband, by changing the LO frequency. Since we record voltage amplitude and phase of the complex baseband signal, a software correction can be made (in the frequency domain) for limited sideband separation due to component mismatch. We verified the complex mixer performance at intervals across the band using an HP 8508A vector voltmeter. Typical sideband separation was $25 \mathrm{~dB}$; for a given frequency, this can be improved to $>30 \mathrm{~dB}$ by careful matching of components and cable lengths.

Changing the bandwidth at baseband is achieved by replacing the low-pass filters. In keeping with our goal of hardware simplicity, we opted to replicate the complex downconverter, changing bandwidth with a cable change on the front of the downconverter chassis. For applications requiring more rapid bandwidth switching, externally controlled RF switches could be employed.

Linearity in the analog portion of the receiver chain is important, otherwise the amplitude of strong signals will be 


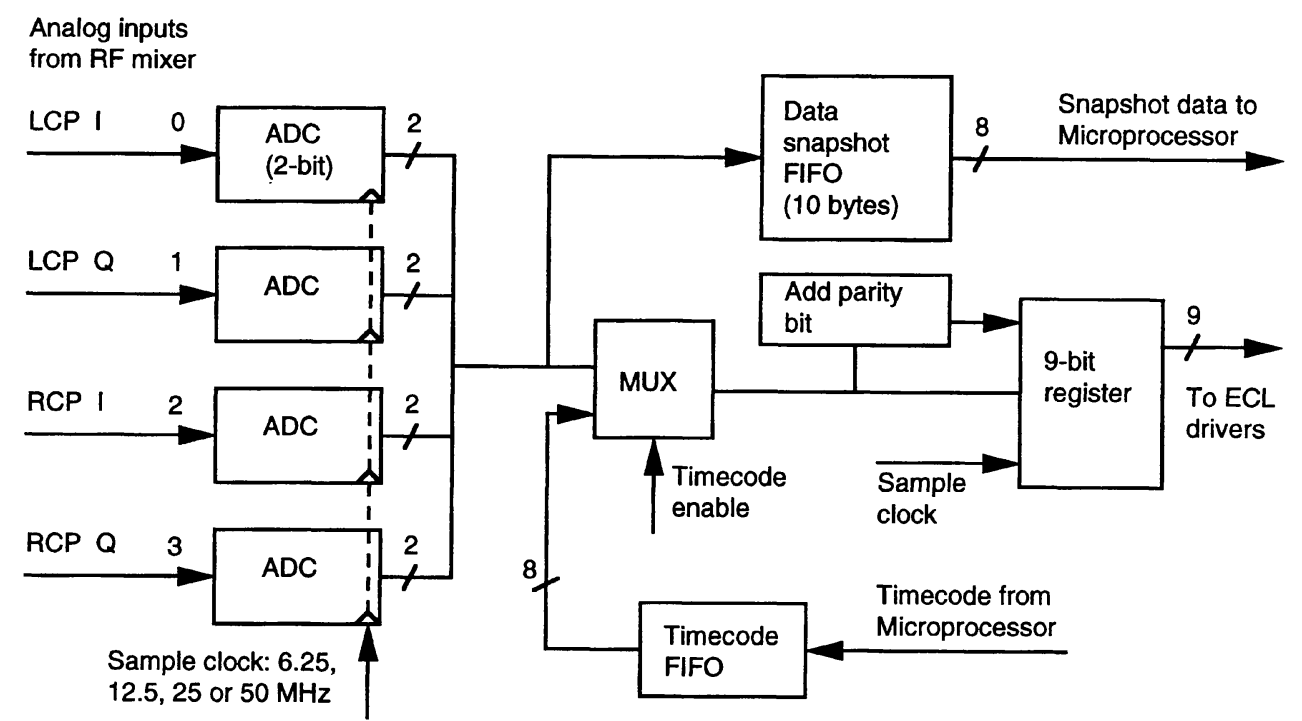

FIG. 2-A simplified block diagram of the functions performed by the custom analog VLSI chip designed for this project.

underestimated, especially if $\mathrm{DM} / P$ is low. At OVRO, we found slight evidence for gain compression in the strong low-DM pulsars we observed: B $1133+16$, B $0950+08$, and B $0329+54$. Such effects are important in studying the statistics of pulse energy distributions, but are not a concern for the test observations presented here.

\subsection{VLSI A/D Converters}

The heart of the digitizer is a custom analog/digital CMOS VLSI chip, mounted on a small wire-wrap board, along with a control microprocessor and CMOS/ECL converters to drive the tape recorder interface. The VLSI chip performs the following functions (see Fig. 2):

(1) 2-bit digitization of four analog input streams from the complex downconverter.

(2) Insertion of synchronization and timing information at regular intervals from the microprocessor.

(3) Parity bit computation and storage in a 9-bit register.

(4) Writing of a 10-byte "snapshot" register (read by the microprocessor for diagnostic purposes).

Combined analog/digital VLSI designs are a relatively recent development, and our chip takes advantage of this capability. This enables almost all high-speed circuitry to be located within the chip. In our implementation, the chip itself is $2.2 \times 2.2 \mathrm{~mm}$, has an element size of $1.2 \mu \mathrm{m}$, contains 4244 CMOS transistors, and resides in a 40 pin package. We selected VLSI rather than discrete components based on: (a) previous experience with similar VLSI-based designs; (b) a trade-off between VLSI design time versus design and layout of many discrete components; and (c) keeping most high-speed logic internal to the chip minimizes matching and crosstalk problems (high-speed discrete logic is limited to the standard ECL interface to the recorder itself).

The most critical function of the entire system, namely
A/D conversion, is accomplished on-chip. Multilevel digitization is achieved using simple but fast comparators. These comparators have $\sim 3$-ns sample time, allowing clock rates up to at least $100 \mathrm{MHz}$. Threshold levels for the comparators are set using a 12-bit D/A converter under microprocessor control. This provides considerable flexibility in how the data are digitized, since relative and absolute levels can be set to high precision. Dynamic compensation for changes in the input power level can be achieved without having to switch the analog attenuation in the RF chain, and minor variations in the characteristics of individual comparators can be accommodated.

Our specific implementation (bandwidth, quantization, number of channels) is matched to the capabilities of the LP-400 tape recorder. We opted to use the fastest record rate, $R=400 \mathrm{Mbit} \mathrm{s}^{-1}$, to take advantage of the widest possible bandwidth. Recording data from two orthogonal polarizations at the Nyquist rate, using 2-bit samples, allows a channel bandwidth of $50 \mathrm{MHz}$. Since we use a complex mixer, the baseband filters pass frequencies in the range -25 to $+25 \mathrm{MHz}$ (Fig. 1).

For the detection of dispersed weak signals (average antenna temperature across the recorded band $\ll$ system temperature) 1-bit sampling makes optimum use of the available storage. For studies of strong pulsars with low $\mathrm{DM} / P$, pulse microstructure with high instantaneous power, or observations in the presence of strong interference, finer quantization is required. The effect of quantization is manifested in a dedispersed profile as a triangular baseline slope with width equal to twice the total dispersion delay across the band (e.g., Navarro 1994). For the WBDR we selected 2-bit sampling (magnitude and sign bits), as a compromise. Up to 4 or 6 bits can be accommodated with straightforward modifications to the current VLSI design, and could be incorporated into a second-generation chip. 


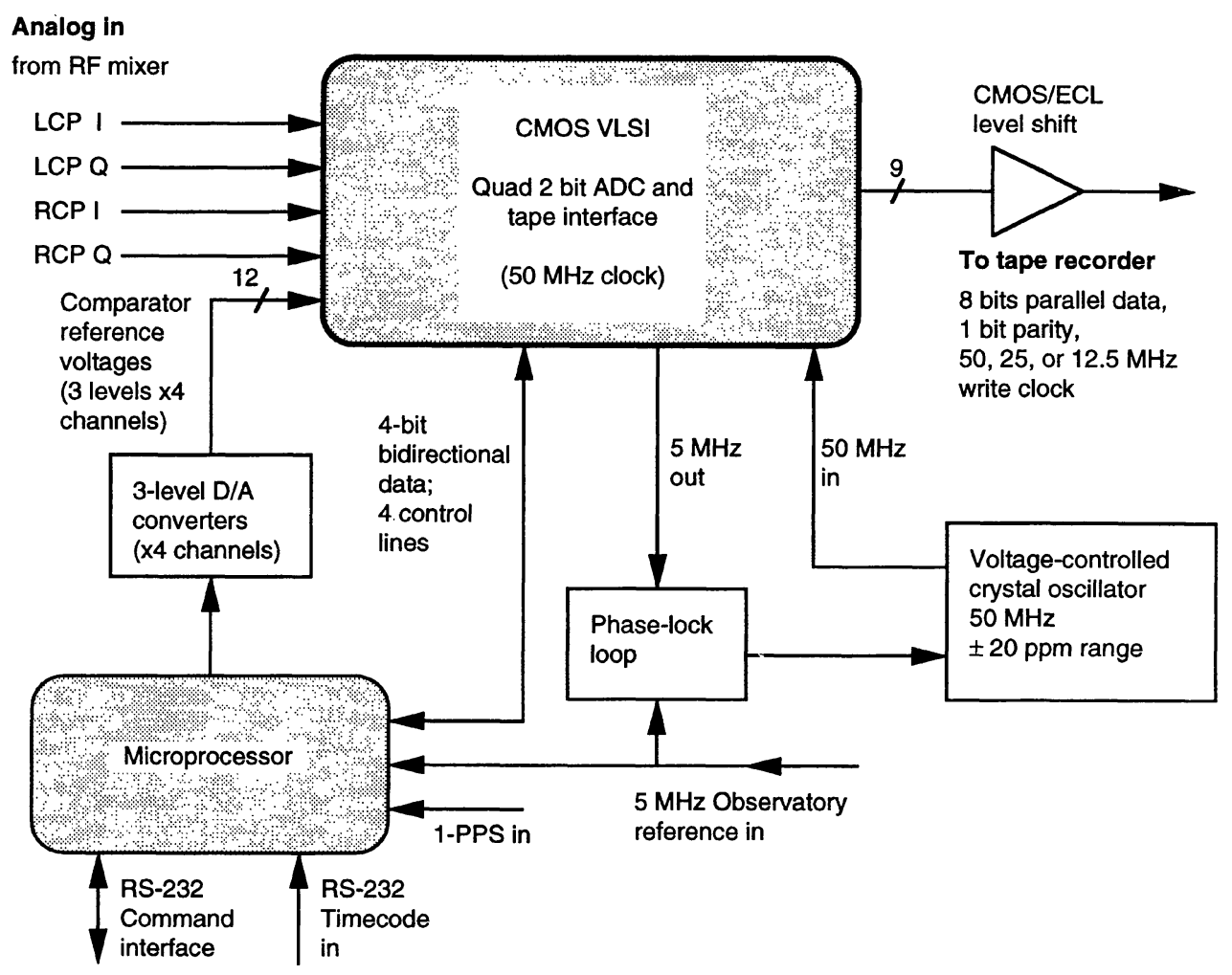

FIG. 3-Block diagram of the functions performed on the digital interface.

\subsection{Digital Section}

The digital interface is a small wire-wrap board which houses the VLSI chip, a Harris RTX 2000 microprocessor, and all the other digital components (Fig. 3). Inputs to the board are (1) analog baseband signals from the downconverter; (2) a $5 \mathrm{MHz}$ observatory reference to phase lock a voltage controlled crystal oscillator which distributes clock signals on the board; (3) a 1 pulse-per-second tick from the observatory time standard to time the placement of synchronization and timing information in the data stream; and (4) an RS-232 command/control port. For convenience of system testing, the digital board can operate without the external 1-PPS and $5 \mathrm{MHz}$ reference signals.

The Harris microprocessor running at $6.25 \mathrm{MHz}$ and using the FORTH language (Sperry 1988) controls several functions of the VLSI chip and provides diagnostic capabilities. Its main functions are (a) set the D/A converter threshold levels, (b) control the optional replacement of 10 bytes of the data stream, at regular intervals, by sync words, and timing information, and (c) read 10-byte "snapshots", of the input data for verification of the system setup.

Sync and timing information are inserted every $20 \mathrm{~ms}$ (set with a parameter in FORTH), via a multiplexer which selects between data from the ADCs and the 10-byte timecode buffer. The time-code buffer comprises: three bytes set to a fixed pattern, for decoding by the analysis program, two bytes for a seconds counter, one for a buffer index counter, and one byte each to store a running mean of the power in each of the four input streams. Analysis software on the parallel computer recovers the timing information (Sec. 3) along with the data. For testing without inputs to the ADCs, data can be continuously replaced by a fixed pattern loaded into the time-code buffer.

Accurate timing of sync words in the data is governed by the input 1-PPS tick and the $5 \mathrm{MHz}$ reference (synchronous with 1 PPS); there is a fixed, measurable offset in the data stream relative to the input tick. The intrinsic relative timing accuracy is $20 \mathrm{~ns}$, and absolute accuracy at the same level is possible if the observatory 1 PPS is known to this level.

In the current implementation, there are four input streams, so with 2-bit sampling, the output data bus is 8-bits wide. The VLSI chip adds a ninth line containing a parity bit. Since this is the configuration required by the Datatape recorder, no buffering of the output data from the VLSI chip is required.

FORTH commands to read and process data from the 10-byte "snapshot" FIFO provide various diagnostic and verification functions. The snapshot FIFO can be read often enough to enable strong (and low $\mathrm{DM} / P$ ) pulsars to be detected in total power; this is accomplished by reading out via the RS-232 port and folding the data in an offline program. This important test at the telescope fully exercises the signal path up to (but not including) the tape recorder itself.

Snapshots are also used to verify the digitizer threshold levels, by accumulating statistics on the level population. For weak signals in Gaussian noise input to a 2-bit digitizer, 
the optimum SNR is obtained with the "magnitude" bit triggered at $1.0 \sigma$, though the SNR function versus input voltage is broad (e.g., Navarro 1994). For our VLSI chip, the nominal input voltage is $100 \mathrm{mV} \mathrm{rms}$, but the operating range is more than a factor of 2 above or below. We used an iterative procedure to set the thresholds on each input; by iterating we assure that the statistics are optimized as well as automatically correcting for minor offsets in the individual comparators. This procedure was performed at the start of each scan (30-60 min duration); changes in total system power were modest during a scan $(\ll 10 \%)$. We chose not to include the refinement of continuous adjustment.

In operation, the Datatape recorder displays diagnostic and status information continuously, including an estimate of the data error rate, using read-after-write. Recordings may be played back at the telescope to verify the presence of data on the tape. Verification of the data integrity currently must await transport of the tapes to the massively parallel computer used for analysis. As described above, a critical test involves a program to perform a byte count between successive sync words. These tests confirm the high data recording fidelity expected from the Datatape recorder.

\subsection{Playback of Recorded Data}

Since the WBDR system generates extremely large datasets, suitable computing hardware and software for data analysis must be considered part of the complete instrument.

The three main parts of the data analysis hardware are the tape recorder, the Intel Paragon (or Delta), and the HIPPI network. HIPPI is the HIgh Performance Parallel Interface, an ANSI standard high-speed data connection used to transfer data between supercomputers, with peak transfer rates of 100 Mbyte $s^{-1}$. This is faster than the maximum rate from the tape recorder (50 Mbyte $\mathrm{s}^{-1}$ ), which means that the data rate is limited by the rate at which data can be distributed and analyzed by the nodes of the parallel computer. A second Datatape LP-400 tape recorder is used to read the data into the Paragon. Data from tape are first read into a 0.5 -Gbyte variable rate buffer, then onto the HIPPI network, where it is read by one of three nodes on the Paragon installed with a HIPPI I/O board. The effective data rate depends on the analysis task being performed, the number of active nodes in the computation, and other input parameters such as FFT lengths.

The analysis programs which read the tape recorder perform several functions on the raw data: (1) search for the 3 -byte sync words in each time-code record, and count the number of bytes between each record; (2) return the first time code in the dataset, and its byte offset from the start; (3) check that successive time codes increment correctly; (4) "unpack" the 2-bit data into floating-point variables. The floating-point data are analyzed as described below.

Experience with reading many tapes (including test tapes with a known fixed bit pattern) confirms that the average bit error rate from the tape is $\sim 10^{-10}$; typically, errors occur not as single incorrect bits, but as bursts of a few to a few tens of bytes. In practice, an error rate this low is negligible for this application in a pulsar astronomy instrument. We have not encountered missed or extra bits, though timing information would be preserved by utilizing the recovered time codes to realign the data array. The next section discusses the software we have developed for analysis of pulsar signals.

\subsection{Computation Hardware}

In order to process the large amount of data collected by the tape recorder, we need a significant amount of computational capability. Our goal is to be able to analyze the data in approximately real time-allowing for the inevitable overhead, this allows a few days of data to be reduced in a reasonable amount of time. An order-of-magnitude calculation (see Sec. 3) shows that we require computation rates of order a few times $10 \mathrm{Gflop} \mathrm{s}^{-1}$ to achieve this goal for a coherent dedispersion analysis of the data. At the Caltech Center for Advanced Computation Research (CACR) we have access to two massively parallel supercomputers with peak speeds comparable to this computation rate: the 512 processor Intel Touchstone Delta with a peak rate of 25 Gflop s ${ }^{-1}$, and a 512-processor Intel Paragon XPS L38 (30 Gflop $s^{-1}$ ).

Efficient single-processor FFT routines can achieve effective speeds of order 40 Mflops. We can attain a total rate of order 20 Gflops by using all 512 nodes of the Intel Touchstone Delta or the Intel Paragon XPS. Accounting for both the FFT calculations and the numerous ancillary operations of data transfer and manipulation, we find that we can perform a coherent dedispersion analysis of the data at an effective rate of about $1 / 2$ "real time" for data collected in the dual polarization mode over $50 \mathrm{MHz}$ of bandwidth.

\section{DATA REDUCTION AND ANALYSIS}

\subsection{Algorithms}

The algorithms used to analyze the data may be divided into two categories: pre-detection and post-detection processing. The first category contains those algorithms which take the raw voltage data and turn it into detected power data; this includes any form of digital filtering. The second category contains the algorithms used to search for and study pulsar signals after signal power has been computed. These analysis algorithms were implemented on the paralle! computers using the object-oriented language $\mathrm{C}++$.

\subsubsection{Incoherent Filterbank}

The simplest pre-detection algorithm is the "incoherent filterbank." This procedure takes a band-limited (bandwidth $B$ ), complex sampled time series and divides it into $n$ real-time series, or channels, wherein each channel is a measure of the total power of the signal within a small band $\Delta f=B / n$. This is the software analog of a hardware filterbank system. In the incoherent filterbank, we simply compute the power spectrum of adjacent small-time segments. This method is illustrated in Fig. 4(a,b). If we have a total of $N$ complex points, we find the power 


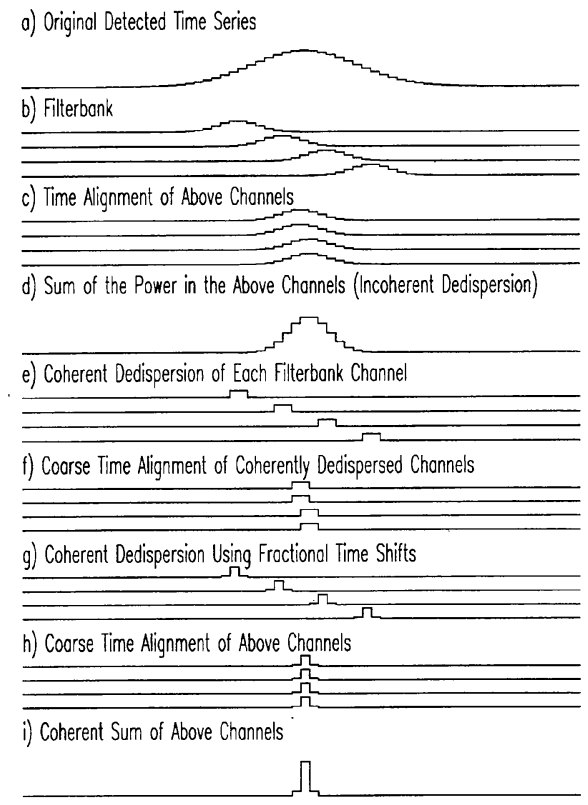

FIG. 4-A schematic representation of the operations performed on a complex-sampled time series in order to dedisperse the pulsar signal.

spectrum of the first 0 to $n-1$ points, then the spectrum of the next $n$ to $2 n-1$ points and so on. The frequency resolution increases with increasing $n$ at the expense of the time resolution, which is decreasing as $1 / n$.

The most efficient way to estimate the power spectrum of $n$ complex points is to perform a fast Fourier transform (FFT) on the $n$ points and then take the squared moduli of each resulting complex point. If we start with $n$ (complex) voltages, we will end up with $n$ (real) frequency channels.

Consider a specific example of the above procedure. Take $100 \mathrm{sec}$ of data from the tape recorder, quadrature sampled at $t_{\text {sam }}=20 \mathrm{~ns}$; this corresponds to $N=5 \times 10^{9}$ samples of complex data (in each polarization). If we require $n=128$ channels each with a bandwidth of $50 \mathrm{MHz} /$ $128=391 \mathrm{kHz}$, we calculate $N / n=3.91 \times 10^{7}$ adjacent, nonoverlapping $n$-point power spectra. Each of the 128 time series has a resolution of $128 t_{\text {sam }}=2.56 \mu \mathrm{s}$. We currently lose SNR by neglecting to overlap the time segments. This refinement will be implemented in a later version of the software.

The incoherent filterbank, like its hardware analog, has limitations. As one increases the number of channels to reduce the effects of dispersion, one simultaneously decreases the time resolution. Hence, for a given DM, there exists an optimum number of channels:

$$
n_{\text {opt }}=2.88\left(\frac{\mathrm{DM}}{\mathrm{pc} / \mathrm{cm}^{3}}\right)^{1 / 2}\left(\frac{B}{\mathrm{MHz}}\right)\left(\frac{f_{c}}{\mathrm{GHz}}\right)^{-3 / 2}
$$

and a corresponding minimum time resolution $n_{\text {opt }} t_{\mathrm{sam}}$. Here, $f_{c}$ is the center frequency of the observing band. To remove dispersion and achieve the intrinsic time resolution of quadrature data, sampled at rate $R\left[\left(t_{\mathrm{sam}}=1 /(2 R)\right]\right.$, we must perform the coherent dedispersion technique described below.

\subsubsection{Coherent Dedispersion}

This method takes recorded voltage signals and filters them in precisely the opposite way that the interstellar medium filters a signal emitted by the pulsar. We model the interstellar medium as a linear filter with the following transfer function (see Appendix B):

$$
H\left(f_{0}+f\right)=\exp \frac{i 2 \pi D f^{2}}{f_{0}^{2}\left(f_{0}+f\right)},
$$

where $f_{0}$ is the center frequency of the band, and $f$ is the baseband frequency (both in $\mathrm{Hz}$ ). The Fourier transform $S\left(f_{0}+f\right)$ of the received signal $s(t)$ is related to the Fourier transform $I\left(f_{0}+f\right)$ of the intrinsic signal $i(t)$ via:

$$
S\left(f_{0}+f\right)=H\left(f_{0}+f\right) I\left(f_{0}+f\right) .
$$

The coherent dedispersion algorithm calculates $I$ by multiplying $S$ by $H^{-1}$ and then transforms $I$ into a real signal $i(t)$ with a time resolution of $1 /(2 R)$. See Appendix A for more details.

This method is applicable when the observation in question allows a dataset of length twice the characteristic broadening time across the receiver band, $2 \Delta t_{b}$, to be stored in the computer's local memory (or the memory of individual processors of a parallel machine). $\Delta t_{B}$ is given by

$$
\left.\Delta t_{B}\left(f_{u}, f_{l}\right)=4.15\left(\frac{\mathrm{DM}}{\mathrm{pc} / \mathrm{cm}^{3}}\right)\left[\frac{f_{l}}{\mathrm{GHz}}\right)^{-2}-\left(\frac{f_{u}}{\mathrm{GHz}}\right)^{-2}\right] \mathrm{ms},
$$

where $f_{u}$ and $f_{l}$ are the frequencies of the upper and lower band edges. Observations at low frequency, wide bandwidth, or large DM require more memory than is typically available. Since one loses computational efficiency when individual processors of a parallel machine have to communicate with each other during the actual computation, we have developed a method which we term "Coherent Filterbank," which greatly reduces the per-node storage requirements. Datasets that have a large $\Delta t_{B}$ can be dedispersed without using multinode or out-of-core Fourier transform methods.

\subsubsection{Coherent Filterbank}

In a coherent filterbank, the dataset is divided into $n$ frequency channels by FFTing adjacent $n$ point blocks of data. Unlike the incoherent filterbank, the power is not detected and each channel is coherently dedispersed separately and then reassembled into one long time series. The bandwidth of each channel is $B / n$ where $B$ is the bandwidth of the original time series. The dispersion smearing time within one of the $n$ time series is about a factor of $n$ smaller than the dispersion smearing time within the original dataset. Compared to coherent dedispersion, the coherent filterbank algorithm requires a factor of $n$ fewer points loaded into memory.

The steps in this algorithm are illustrated in Fig. 4. First, calculate adjacent $n$ point FFTs of the data. This results in $n$ complex time series each with a length of $m / n$ where $m$ is the total number of complex samples loaded into 
memory $\left(m \geqslant B \Delta t_{B} / n\right)(4 \mathrm{a}, \mathrm{b})$. Next, the $n$ small time series are coherently dedispersed $(4 \mathrm{e})$. This will remove the dispersion broadening within a channel, but it will not remove the arrival time delays between different channels. The arrival time delays may be partially removed by shifting the time origin of each channel by an appropriate amount (4f). This method of time shifting is also used when incoherently dedispersing a set of filterbank channels (4c). Incoherent dedispersion is described in more detail in Sec. 3.1.4. Time shifting alone will not completely remove the arrival time delays since the time resolution of each time series $\left(n t_{\mathrm{sam}}\right)$ is relatively coarse. Using a transfer function of the form

$$
H(f) \exp [i 2 \pi f \delta t(n)]
$$

coherently to dedisperse each channel will remove that part of the time delay which is less than the time resolution $(4 \mathrm{~g})$. $H(f)$ is the usual ISM transfer function and $\delta t(n)$ is the necessary time delay for a given channel $n$. Once this correction is made, the remaining arrival time delays can be removed using time shifting methods (4h).

At this point, we have a set of adjacent dedispersed filterbank channels. If we are interested in the frequency spectrum of the signal, we can stop here, detect total power, and move on to post-detection processing. On the other hand, we may be able to obtain a dedispersed time series with a time resolution of $1 / 2 B$ by inverse fast Fourier transforming each set of $n$ channels after the arrival time delays have been removed. This last step has not yet been proven in practice.

\subsubsection{Post-Detection Processing}

The above pre-detection algorithms produce either one dedispersed time series or a collection of time series representing $n$ filterbank channel outputs. These results can be analyzed using standard pulsar analysis techniques. Software packages such as PSRPACK (Deich 1996) and TEMPO (Taylor and Weisberg 1989) are used for this purpose.

One standard technique called incoherent dedispersion removes arrival time delays from a set of filterbank channels and then computes one single dedispersed time series. The value of the dedispersed time series at time $t$ is obtained by adding together the power across the $n$ channels at time $t$.

Each filterbank channel has a well-defined center frequency $f_{c}$. Due to the nature of a cold plasma, $\Delta t_{B}\left(f_{c 1}, f_{c 2}\right)$ given by Eq. (4) also equals the pulse arrivaltime difference between two channels with center frequencies $f_{c 1}$ and $f_{c 2}$, respectively. If we fix $f_{c 2}$ equal to some fiducial frequency, $f_{o}$, then we can shift the time origin of each filterbank channel by $\Delta t_{B}\left(f_{c}, f_{o}\right)$. After performing this arrival time correction, the channels are added together to form one dedispersed time series (see Fig. $4 a-d$ ).

\subsection{Computation Rates}

In this section, we estimate the number of floating point operations required by each pre-detection algorithm. Given these estimates, we calculate the computational speed re-
TABLE 1

Operations Count and Real Time Computation Rates

\begin{tabular}{|c|c|c|}
\hline Algorithm & $\begin{array}{c}\text { Number of } \\
\text { operations } \\
\text { per polarization }{ }^{\mathrm{a}}\end{array}$ & $\begin{array}{c}\text { Real time } \\
\text { computation rate fo } \\
\text { two polarizations }\end{array}$ \\
\hline Filterbank (no overlap) & $5 N \log _{2}(n)+3 N$ & 2.65 GFlops \\
\hline Coherent dedispersion & $10 N \log _{2}(m)+7 N$ & 10.35 GFlops \\
\hline $\begin{array}{l}\text { Coherent filterbank: } \\
n \text { channel output }\end{array}$ & $10 N \log _{2}(m)+7 N-5 N \log _{2}(n)$ & 7.85 GFlops \\
\hline $\begin{array}{l}\text { One dedispersed } \\
\text { time series }\end{array}$ & $10 N \log _{2}(m)+7 N$ & 10.35 GFlops \\
\hline
\end{tabular}

${ }^{a} N$ is the total number of complex points in the time series, $m$ is the number of complex points loaded into a given nodes memory at a given time, and $n$ is the number of channels produced by the algorithm.

${ }^{\mathrm{b}}$ The real-time rates where estimated for a data collection rate of $50 \mathrm{MHz}$, $n=2^{10}$, and $m=2^{20}$.

quired to analyze a given dataset in real time. For all calculations below, $N$ is the total number of complex data points, $m$ is the number of complex samples loaded into a node's memory at a given time, and $n$ is the resulting number of individual time series or filterbank channels.

One FFT of length $n$ requires $5 n \log _{2}(n)$ floating point operations. Power detection across all $n$ channels requires $3 n$ operations. An $n$ channel software filterbank performs both of these computations $N / n$ times. Hence, the total number of floating point operations performed by an $n$ channel filterbank is:

$$
\frac{N}{n}\left[5 n \log _{2}(n)+3 n\right]=5 N \log _{2}(n)+3 N .
$$

The coherent dedispersion method calculates one forward FFT, multiplies the result by the transfer function, calculates one inverse FFT, and finally detects power. Since it does this $\mathrm{N} / \mathrm{m}$ times, coherent dedispersion requires a total of

$$
\frac{N}{m}\left[10 m \log _{2}(m)+7 m\right]=10 N \log _{2}(m)+7 N
$$

floating points operations. The coherent filterbank algorithm takes the $m$ points and calculates $m / n$ FFTs of length $n$. This takes $5 m \log _{2}(n)$ operations. Coherently dedispersing each channel takes a total of $10 m \log _{2}(m / n)+6 n$ operations. Performing these operations and detecting power $\mathrm{N} / \mathrm{m}$ times gives:

$$
10 N \log _{2}(m)+7 N-5 N \log _{2}(n)
$$

as the total number of operations required by the coherent filterbank if one wants $n$ coherently dedispersed filterbank channels. If the required output is a dedispersed time series, then $m / n \times N / m$ additional $n$ point FFTs must be calculated. The total number of operations (including power detection) becomes:

$$
10 N \log _{2}(m)+7 N \text {. }
$$

The above results are summarized in Table 1 . The realtime computation rate is the rate needed to process the data within a time equal to the time spent acquiring the data. The computations rates quoted in Table 1 are for two polariza- 


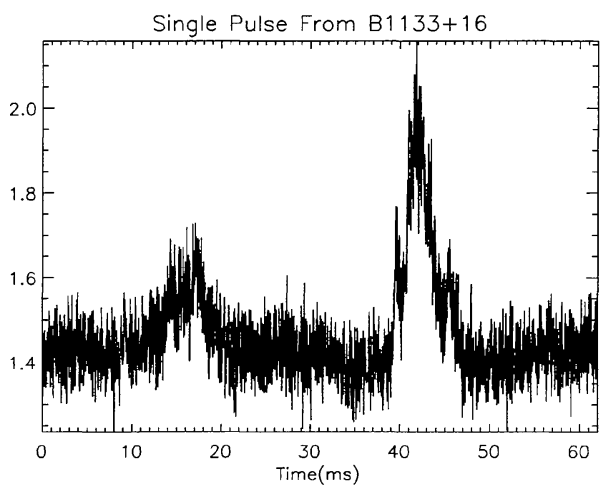

FIG. 5-Single pulse from B $1133+16$ at $600 \mathrm{MHz}$ corrected for dispersion with a 1024-channel software filterbank and $\mathrm{DM}=4.8471 \mathrm{pc} \mathrm{cm}^{-3} . \mathrm{Ob}$ servation from JD 2449652.25 of the strongest pulse seen in a $730 \mathrm{~s}$ scan. Time sampling is $20.48 \mu \mathrm{s}$.

tions sampled at the highest data collection rate of $50 \mathrm{MHz}$. Where applicable, $n=2^{10}$, and $m=2^{20}$.

\subsection{Polarization Processing}

Since we record two orthogonal polarizations we have the ability to calculate all four Stokes parameters. The current version of the analysis software sums total power collected by both polarizations, yielding Stokes "II." For the first observations with the WBDR at OVRO (Sec. 4), we did not perform a calibration of the receiver channels required for the remaining Stokes parameters. However, in the future we will record calibration scans which will enable a complete polarization solution, following the method of Stinebring 1982.

\section{FIRST OBSERVATIONS}

The first observations using the WBDR system were made at the 40-m telescope at the Owens Valley Radio Observatory, in 1994 October. We used a dual circular feed and room-temperature FET receiver, with a zenith system temperature of $\approx 120 \mathrm{~K}$. The system sensitivity is $\sim 0.15$ $\mathrm{K} \mathrm{Jy}^{-1}$, with no significant variation with zenith angle. The system was described in Sec. 2.1 (Fig. 1). We observed a variety of objects during these test observations, including millisecond pulsars, strong normal pulsars, and globular clusters. To illustrate the performance of the WBDR system, we show results from applying, respectively, a software filterbank and coherent dedispersion to observations of two strong pulsars, PSR B $1133+16$, and the Crab Nebula pulsar (PSR B 0531+21).

\subsection{PSR B 1133+16}

The pulsar PSR B $1133+16$ is a strong, old $\left(P=1.1879 \mathrm{~s}\right.$, characteristic age $\left.P /(2 \dot{P})=5 \times 10^{6} \mathrm{yr}\right)$ pulsar which exhibits nulling (Ritchings 1976). Since this pulsar has a low DM we can achieve high time resolution with a software filterbank, Eq. (1). Figure 5 shows the largest single pulse observed in our OVRO observations. Microstructure is visible on scales down to the sample time

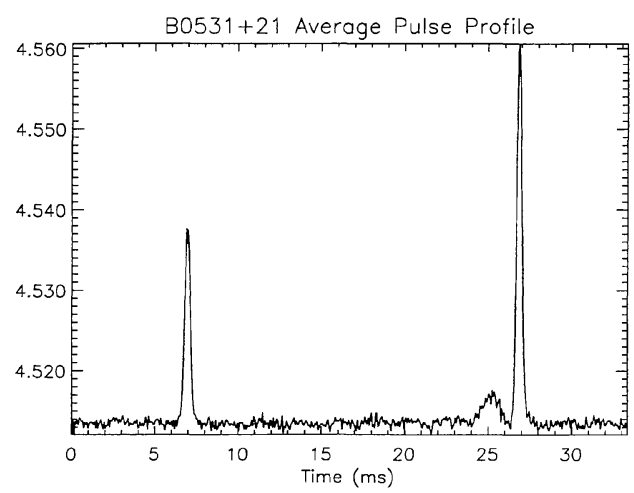

FIG. 6-Mean pulse profile of B $0531+21$ in Stokes "I', at $600.0 \mathrm{MHz}$ corrected for dispersion using $\mathrm{DM}=56.811 \mathrm{pc} \mathrm{cm}^{-3}$. Scan length $860 \mathrm{~s}$ starting at JD 2449652.19. Time sampling is $65.2 \mu \mathrm{s}$; DM smearing is $52.7 \mu$ s per filterbank channel. The amplitude scale is arbitrary.

of the filterbank, $20.48 \mu \mathrm{s}$, or $<1.7 \times 10^{-5}$ of the pulse period. The peak amplitude in this pulse is $\sim 40 \times$ that of the mean profile. This is not surprising, given the extreme pulse-to-pulse variations known in this pulsar (Cordes and Hankins 1977).

\subsection{Crab Pulsar}

The Crab pulsar is one of the brighter pulsars we observed, and since it is also highly dispersed we made it a test case for coherent dedispersion. At $600 \mathrm{MHz}$ smearing $\Delta t$ across our $50 \mathrm{MHz}$ band is $101 \mathrm{~ms}$, or about three pulse periods. Using a software filterbank with 2048 channels and 512 phase bins, we obtained a mean pulse profile having a time resolution of $65.3 \mu \mathrm{s}$. The DM time smearing per channel is $52.7 \mu \mathrm{s}$. This profile is shown in Fig. 6. The main pulse, interpulse, and precursor relative amplitudes agree with published profiles (Lyne and Thorne 1975; Moffett and Hankins 1996).

Giant pulses are a well-known phenomenon in the Crab pulsar (Lundgren et al. 1995); their high SNR relative to the mean enables us to probe microstructure on time scales much less than the bin width in Fig. 6. We took the strongest giant pulse observed in the same 860 -s scan as a "test case" for coherent dedispersion (Fig. 7). Note that the SNR throughout the "on-pulse" period is substantially greater than unity, even at 10 -ns resolution.

We studied the frequency structure of this giant pulse by applying a software filterbank with eight channels to the dedispersed time series, which degrades the time resolution only by a factor of 8 , in contrast to Eq. (1), (see Fig. 8). From this small filterbank, we confirmed that the DM of this pulse is consistent with that of the mean profile for the scan.

\section{CONCLUSIONS}

We have presented a description of a new data acquisition system for pulsar astronomy. This system has proved to be powerful and versatile, and will be used in a variety of pulsar projects. The ability to dedisperse pulsar signals to a time resolution of $1 /(2 B)=10$ ns has allowed detailed 


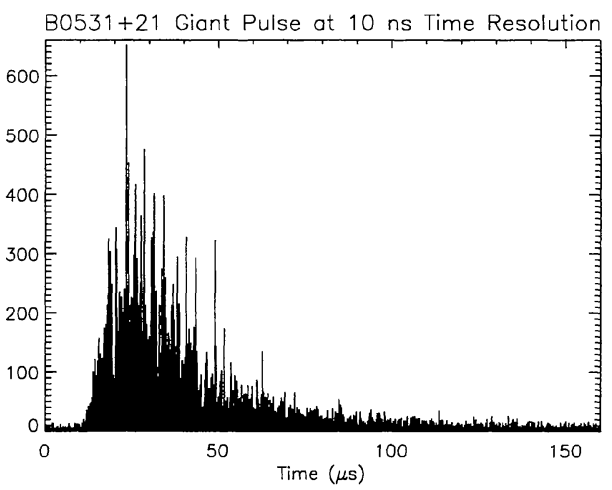

FIG. 7-Giant pulse from B $0531+21$ at $600 \mathrm{MHz}$ corrected for dispersion using $\mathrm{DM}=56.811 \mathrm{pc} \mathrm{cm}^{-3}$. Observations from JD 2449652.19, and represents the strongest pulse seen in the $838 \mathrm{~s}$ scan. Sample time is $10 \mathrm{~ns}$. The amplitude scale is arbitrary.

study of pulse microstructure and nanostructure, and may lead to better understanding of the fundamental pulsar emission processes.

The first use of the WBDR system for pulsar astronomy was on the 64-m telescope at Parkes Observatory in 1995 July. We observed globular clusters and selected galacticplane pulsars at 430,660, and $1380 \mathrm{MHz}$. The results of these investigations will be published elsewhere.

A number of future directions for the instrument are possible. We will use it with the upgraded 305-m Arecibo Observatory for deep searches for millisecond pulsars in globular clusters. With minor modification to operate in a "burst mode," the acquisition system could study microstructure and giant pulses with a much wider bandwidth, and finer voltage quantization.

While the system used at Parkes Observatory had adequate capability for verifying the equipment setup (Sec. 2), the ability to analyze tape data in near real time was lacking. We have developed a workstation interface to the Datatape LP-400 recorder which takes advantage of the recorder's ability to play back at $1 / 8$ speed, or 6.25 Mbyte $\mathrm{s}^{-1}$. Using a 16-bit parallel interface card in a Sun Microsystems workstation, we have demonstrated that large datasets can be read from tape. In future observing sessions, this capability will allow better system verification, as well

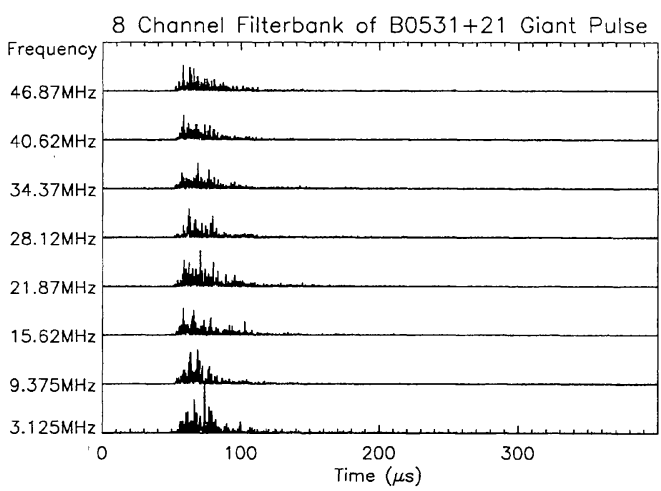

FIG. 8-An eight channel filterbank of the largest giant pulse found in an $838 \mathrm{~s}$ observation of $\mathrm{B} 0531+21$. The time resolution is $80 \mu \mathrm{s}$. as the ability to do science analysis at the telescope.

We are grateful to Stuart Anderson and Vicky Kaspi for their help in implementing the coherent dedispersion algorithm, and comments on the text. John Yamasaki, Derrick Key, Mark Hodges, and Russ Keeney helped with hardware construction. We thank Shri Kulkarni, José Navarro, and Jagmit Sandhu for many stimulating discussions. Datatape Inc., provided advice and engineering support throughout the development of this system. This work was supported by the NSF under Grant Nos. AST 90-20787 and ASC 9318145.

\section{APPENDIX A: ANALYSIS OF QUADRATURE (COMPLEX) SAMPLED DATA}

In the first part of this Appendix, we will show that the output of a complex mixer is equivalent to multiplying the real signal, $x(t)$, by $e^{i \omega_{0} t}$ and then low pass filtering the result. We next describe how one converts a complex signal sampled at a rate of $R$ into a real signal sampled at a rate of $2 R$.

The mixer described in Sec. 2 takes $x(t)$ and creates the following two signals:

$$
\begin{aligned}
& I(t)=x(t) \cos \left(\omega_{0} t+\phi\right), \\
& Q(t)=x(t) \sin \left(\omega_{0} t+\phi\right),
\end{aligned}
$$

where $\phi$ can be taken to be zero without loss of generality. These signals are then filtered with a low-pass filter to remove frequencies outside the range $-\Delta B / 2-\Delta B / 2$ :

$$
\begin{aligned}
& I_{b}(t)=\int x\left(t^{\prime}\right) \cos \left(\omega_{0} t^{\prime}\right) B\left(t-t^{\prime}\right) d t^{\prime}, \\
& Q_{b}(t)=\int x\left(t^{\prime}\right) \sin \left(\omega_{0} t^{\prime}\right) B\left(t-t^{\prime}\right) d t^{\prime},
\end{aligned}
$$

where $B(t)$ is the filter transfer function in the time domain and the subscript $b$ denotes band-limited. If we define $y(t)$ and $y_{b}(t)$ such that

$$
\begin{gathered}
y(t)=I(t)+i Q(t)=x(t) e^{i \omega_{0} t}, \\
y_{b}(t)=I_{b}(t)+i Q_{b}(t),
\end{gathered}
$$

then Eqs. (A3) and (A4) imply

$$
\begin{aligned}
y_{b}(t) & =\int x\left(t^{\prime}\right) e^{i \omega_{0} t^{\prime}} B\left(t-t^{\prime}\right) d t^{\prime} \\
& =\int y\left(t^{\prime}\right) B\left(t-t^{\prime}\right) d t^{\prime},
\end{aligned}
$$

i.e., the output of the mixer can be represented as a complex number, $y_{b}(t)$, that was created by first calculating $y(t)=x(t) e^{i \omega_{0} t}$ and then filtering the result with the filter represented by $B(t)$.

By taking the Fourier transform of this equation, we can understand the frequency content of the complex signal. The forward Fourier transform of $y_{b}(t)$ is defined as:

$$
\widetilde{y_{b}}(\omega)=\frac{1}{2 \pi} \int y_{b}(t) e^{i \omega t} d t .
$$

Using the convolution theorem twice, we obtain: 


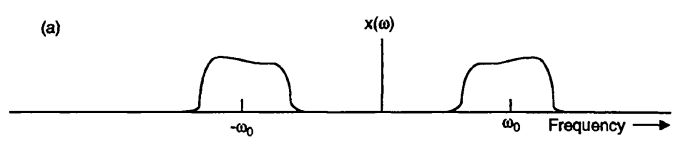

(b) $\quad x\left(\omega+\omega_{0}\right)$
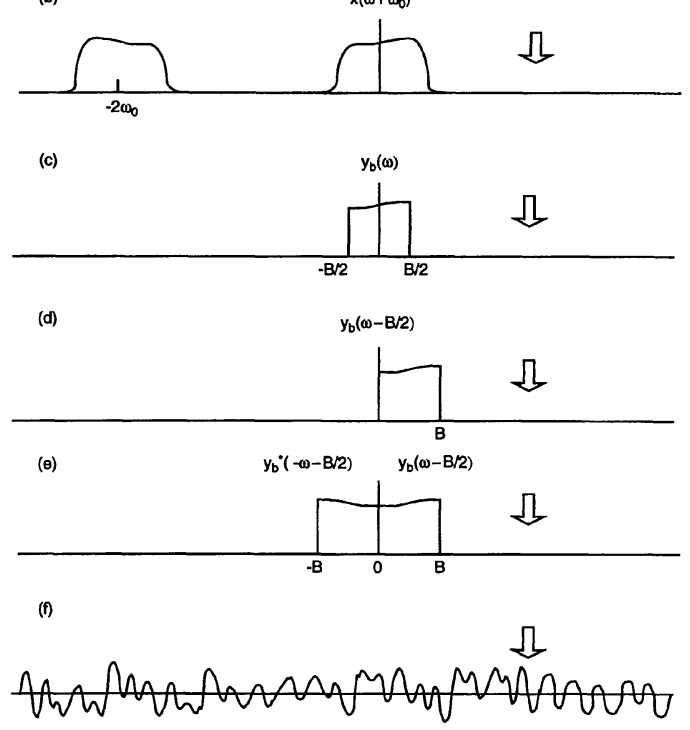

FIG. 9-A representation of the operations performed on the RF signal by the analog and digital hardware, and the conversion from quadrature (complex) sampling to real sampling.

$$
\begin{aligned}
\widetilde{y}_{b}(\omega) & =\widetilde{B}(\omega) \int \widetilde{x}\left(\omega^{\prime}\right) \delta\left(\omega+\omega_{0}-\omega^{\prime}\right) d \omega^{\prime} \\
& =\widetilde{B}(\omega) \widetilde{x}\left(\omega+\omega_{0}\right),
\end{aligned}
$$

$y_{b}(t)$ contains the information of $x(t)$ within a frequency band $\Delta B$ centered about $\omega_{0}$. Figure 9 graphically displays the relationship between $\widetilde{x}(\omega)$ and $\widetilde{y_{b}}(\omega)$ and the operations described below.

Next, take a complex time series $y_{b}(t)$ sampled at the Nyquist rate $R$ which is equal to the bandwidth $B$ of the complex signal. (If $y_{b}$ were real, the Nyquist rate would be $2 B$.) We want to take the complex time series and calculate a real baseband time series $x_{b}(t)$ Nyquist sampled at a rate of $2 B$. This is efficiently achieved by taking the discrete Fourier transform of the signal and then setting

$$
\tilde{x}_{b}(\omega)=\left\{\begin{array}{cc}
\tilde{y}_{b}(\omega-B / 2) & \text { for } \omega \geqslant 0 \\
\tilde{y}_{b}^{*}(-\omega-B / 2) & \text { for } \omega<0
\end{array}\right.
$$

$\widetilde{x_{b}}(\omega)$ now represents the discrete Fourier transform of a real baseband signal with bandwidth $B$ since $\tilde{x}_{b}(-\omega)=\widetilde{x}_{b}^{*}(\omega)$ and $\omega$ ranges from $-B$ to $B$ [Fig. 9(e)]. The inverse discrete Fourier transform of $\tilde{x}_{b}(\omega)$ will be a real-time series sampled at the Nyquist rate of $2 B$. Note that this last step changes the representation, but preserves the information content of the signal: $N$ complex samples are now $2 N$ real samples.

\section{APPENDIX B: THE INTERSTELLAR MEDIUM TRANSFER FUNCTION}

In this Appendix we derive the form of the interstellar medium (ISM) transfer function appropriate for our complex-sampled data. If $S(t)$ is the intrinsic real signal emitted by the pulsar, then the Fourier transform of the signal $x(t)$ just before the complex mixer will be

$$
\widetilde{x}(\omega)=\widetilde{S}(\omega) \widetilde{H}(\omega) \widetilde{R}(\omega) .
$$

$\widetilde{H}(\omega)$ is the ISM transfer function and $\widetilde{R}(\omega)$ is the telescope transfer function. From Eq. (A10), the band limited signal after the mixer has the following Fourier transform:

$$
\widetilde{y_{b}}(\omega)=\widetilde{S}\left(\omega+\omega_{0}\right) \widetilde{H}\left(\omega+\omega_{0}\right) \widetilde{R}\left(\omega+\omega_{0}\right) \widetilde{B}(\omega) .
$$

In order to remove the effects of the ISM, we simply multiply $\widetilde{y}_{b}(\omega)$ by $\widetilde{H}^{-1}\left(\omega+\omega_{0}\right)$.

$\widetilde{H}(\omega)$ has the following form:

$$
\begin{gathered}
\tilde{H}(\omega)=\exp \left(i \frac{\omega n(\omega) x}{c}\right), \\
n(\omega)=\sqrt{1-\frac{\omega_{p}^{2}}{\omega^{2}}}, \\
\omega_{p}^{2}=\frac{\left\langle n_{e}\right\rangle e^{2}}{m_{e}},
\end{gathered}
$$

where $n(\omega)$ in the index of refraction of a cold plasma, $\omega_{p}$ is the plasma frequency, $\left\langle n_{e}\right\rangle$ is the average electron number density, $e$ is the electron charge, and $m_{e}$ is the electron mass. Since $\omega_{p} \sim 10^{5} \mathrm{rad} \mathrm{s}^{-1}$ and we observe with typical center frequencies of order $10^{10} \mathrm{rad} \mathrm{s}^{-1}$, we can expand $n(\omega)$ in a Taylor series and keep only those terms of order $\left(\omega_{p}^{2} / \omega^{2}\right)$ and lower. $\widetilde{H}(\omega)$ then becomes:

$$
\widetilde{H}(\omega)=\exp \left(\frac{i \omega x}{c}-\frac{i \omega_{p}^{2} x}{2 \omega c}\right) .
$$

Following Hankins and Rickett 1975, we define:

$$
\frac{\omega_{p} x}{2 c}=(2 \pi)^{2} D=\frac{(2 \pi)^{2} D M 10^{16}}{2.410000},
$$

where DM is the dispersion measure in $\mathrm{pc} \mathrm{cm}^{-3}$. Substituting for $\omega_{p}$ in (B6), we obtain:

$$
\widetilde{H}\left(\omega+\omega_{0}\right)=\exp \left(\frac{i\left(\omega+\omega_{0}\right) x}{c}\right) \exp \left(-\frac{i(2 \pi)^{2} D}{\omega+\omega_{0}}\right) .
$$

Using the algebraic relationship:

$$
\frac{1}{\omega+\omega_{0}}=\frac{\omega^{2}}{\left(\omega+\omega_{0}\right) \omega_{0}^{2}}-\frac{1}{\omega_{0}}+\frac{\omega}{\omega_{0}^{2}}
$$

we obtain: 


$$
\begin{aligned}
\widetilde{H}\left(\omega+\omega_{0}\right)= & \exp \left[i \frac{\omega_{0} x}{c}+i \frac{(2 \pi)^{2} D}{\omega_{0}}\right. \\
& \left.+i \omega\left(\frac{x}{c}-\frac{(2 \pi)^{2} D}{\omega_{0}^{2}}\right)\right] \\
& \times \exp \left(-i \frac{(2 \pi)^{2} D \omega^{2}}{\left(\omega+\omega_{0}\right) \omega_{0}^{2}}\right) .
\end{aligned}
$$

Transfer functions of the form $e^{i \alpha}$ have the effect of rotating the complex phase of each time sample by $\alpha$. Transfer functions of the form $e^{i=\beta \omega}$ merely shift the time origin of the series by $\beta$. Neither of these operations effect the results of any of our current analysis techniques, and therefore we can simply remove them from the ISM transfer function. Our final result is:

$$
\widetilde{H}\left(\omega+\omega_{0}\right)=\exp \left(-i \frac{(2 \pi)^{2} D \omega^{2}}{\left(\omega+\omega_{0}\right) \omega_{o}^{2}}\right) .
$$

\section{REFERENCES}

Backer, D. C. 1988, in Radio Wave Scattering in the Interstellar Medium, AIP Conference Proceedings, No. 174, ed. J. M. Cordes, B. J. Rickett, and D. C. Backer (New York, American Institute of Physics), p. 111

Backer, D. C. 1995, in Pulsar Science with the Upgraded Arecibo Telescope, 27-28 October, 1995

Bhattacharya, D. 1992, in X-ray Binaries and Recycled Pulsars, ed. E. P. J. van den Heuvel and S. A. Rappaport (Dordrecht, Kluwer), pp. 257-268

Cordes, J. M., and Hankins, T. H. 1977, ApJ, 218, 484

Cordes, J. M., Weisberg, J. M., and Boriakoff, V. 1985, ApJ, 288, 221

Deich, W. 1996, Ph.D. thesis, California Institute of Technology Hankins, T. H. 1971, ApJ, 169, 487

Hankins, T. H., and Rickett, B. J. 1975, Meth. Comp. Phys., 14, 55

Hankins, T. H., Stinebring, D. R., and Rawley, L. A. 1987, ApJ, 315,149

Lundgren, S. C., Cordes, J. M., Ulmer, M., Matz, S. M., Lomatch, S., Foster, R. S., and Hankins, T. 1995, ApJ, 453, 433

Lyne, A. G., and Thorne, D. 1975, MNRAS, 172, 97

Moffett, D. A., and Hankins, T. H. 1996, ApJ, 468, 779

Navarro, J. 1994, Ph.D. thesis, California Institute of Technology Phinney, E. S., and Kulkarni, S. R. 1994, ARAA, 32, 591

Ritchings, R. T. 1976, MNRAS, 176, 249

Sperry T. 1988, Embedded Systems Programming, 65-69

Stinebring, D. R. 1982, Ph.D. thesis, Cornell University

Stinebring, D. R., Kaspi, V. M., Nice, D. J., Ryba, M. F., Taylor, J. H., Thorsett, S. E., and Hankins, T. H. 1992, Rev. Sci. Instrum., 63, 3551

Taylor, J. H., and Cordes, J. M. 1993, ApJ, 411, 674

Taylor, J. H., and Stinebring, D. R. 1986, ARAA, 24, 285

Taylor, J. H., and Weisberg, J. M. 1989, ApJ, 345, 434

Wietfeldt, R., Van Straten, W., Del Rizzo, D., Bartel, N., Cannon, W., Bailes, M., Reynolds, J., and Wilson, W. 1995, in Pulsar Science with the Upgraded Arecibo Telescope, 27-28 October, 1995 